\title{
OX40 expression in neutrophils promotes hepatic ischemia/reperfusion injury
}

\author{
Hua Jin, ${ }^{1,2,3,4}$ Chunpan Zhang, ${ }^{2,3,4}$ Chengyang Sun, ${ }^{2,3,4}$ Xinyan Zhao, ${ }^{5}$ Dan Tian, ${ }^{1,2,3,4}$ Wen Shi, ${ }^{1,2,3,4}$ \\ Yue Tian, ${ }^{2,3,4}$ Kai Liu, ${ }^{2,3,4}$ Guangyong Sun, ${ }^{1,2,3,4}$ Hufeng $X u_{,}{ }^{2,3,4}$ and Dong Zhang ${ }^{1,2,3,4,5}$ \\ ${ }^{1}$ Ceneral Surgery Department and ${ }^{2}$ Experimental and Translational Research Center, Beijing Friendship Hospital, Capital \\ Medical University, Beijing, China. ${ }^{3}$ Beijing Clinical Research Institute, Beijing, China. ${ }^{4}$ Beijing Key Laboratory of Tolerance \\ Induction and Organ Protection in Transplantation, Beijing, China. ${ }^{5}$ National Clinical Research Center for Digestive \\ Diseases, Beijing, China.
}

\begin{abstract}
Neutrophils play critical roles during the initial phase of hepatic ischemia/reperfusion injury (HIRI). However, the regulation of neutrophil activation, infiltration, and proinflammatory cytokine secretion has not been fully elucidated. In this study, we revealed that OX40 was expressed by neutrophils, its expression in neutrophils was time-dependently upregulated following HIRI, and Ox40 knockout markedly alleviated liver injury. Compared with wild-type neutrophils, the adoptive transfer of $\mathrm{O} \times 4 \mathrm{O}^{-/-}$neutrophils decreased HIRI in neutrophil-depleted $\mathrm{Rag} 2 / \mathrm{II} 2 \mathrm{rg}^{-/-}$or $\mathrm{O} \times 4 \mathrm{O}^{-/-}$mice. Moreover, consistently, the in vitro experiments showed that $0 \times 40$ not only prolonged neutrophil survival but also promoted proinflammatory cytokines, ROS production, and even neutrophil chemotaxis. Further investigation demonstrated that the knockout of $0 \times 40$ in neutrophils inhibited NF- KB signaling via the TRAF1/2/4 and IKK $\alpha /$ IKK $\beta / / K B \alpha$ pathways. OX40L and $0 X 86$ stimulation could enhance neutrophil activation and survival in vitro and in vivo. In conclusion, our study provides a new understanding of $0 \times 40$, which is expressed not only in adaptive immune cells but also in innate immune cells, i.e., neutrophils, contributing to the activation and survival of neutrophils. These findings provide a novel potential therapeutic target for the prevention of HIRI during liver transplantation or hepatic surgery.
\end{abstract}

Authorship note: HJ and CZ contributed equally to this study.

Conflict of interest: The authors have declared that no conflict of interest exists.

Copyright: (c) 2019, American Society for Clinical Investigation.

Submitted: April 22, 2019

Accepted: September 20, 2019

Published: November 1, 2019.

Reference information: JCI Insight. 2019;4(21):e129736.

https://doi.org/10.1172/jci.

insight.129736.

\section{Introduction}

Hepatic ischemia and reperfusion injury (HIRI) is a major cause of postoperative complications following liver transplantation and hepatic surgery (1). In addition, because the liver is an important immunological organ, diverse immune cells are closely related to the development of HIRI $(2,3)$. Among these effector cells, neutrophils play a critical role during the initial phase of liver injury after reperfusion (4). Activation and infiltration of neutrophils are initiated through ROS and proinflammatory cytokines, which are generated mostly by hepatocytes or Kupffer cells during reoxygenation insult $(5,6)$. HIRI caused by neutrophils is triggered mainly by 2 cytotoxic mediators, i.e., ROS and several proteases (such as elastases, cathepsin $\mathrm{G}$, and myeloperoxidase) (7). Moreover, neutrophils produce proinflammatory cytokines, such as TNF- $\alpha$, IL-1 $\beta$, and IL-17A, that contribute to the inflammatory immune response $(8,9)$.

OX40 (CD134) is widely considered a T cell costimulatory molecule that is time-dependently expressed in activated $\mathrm{CD}^{+} \mathrm{T}$ cells and $\mathrm{CD} 8^{+} \mathrm{T}$ cells (10). OX40 is essential for the $\mathrm{T}$ cell regulation of cell proliferation, survival, differentiation, and even cytokine production (11), which are associated with inflammatory immune activation in many liver diseases, such as hepatitis B (12), hepatocellular carcinoma (13), and concanavalin A-induced hepatitis (14). Interestingly, by performing an in vitro stimulation and analysis of neutrophils from healthy human PBMCs, one group reported that OX40 is constitutively expressed in human peripheral blood neutrophils and regulates neutrophil survival, indicating that OX40 contributes not only to adaptive immunity but also to innate immunity (15). However, OX40 expression in neutrophils in vivo and its roles and underlying mechanisms in pathological disease conditions remain obscure.

In this study, we monitored OX40 expression in neutrophils at different reperfusion times and identified the role of OX40 in neutrophils in HIRI. In addition, we explored whether OX40 expression in neutrophils contributes to the biological functions of neutrophils, including proinflammatory cytokine and ROS production, chemotaxis, and cell survival, via the tumor necrosis factor receptor-associated factor 1/2/4-associated (Traf1/2/4-associated) NF- $\mathrm{BB}$ signaling pathway. 


\section{Results}

HIRI upregulates OX40 expression in neutrophils. To study the role of OX40 molecules in neutrophils in HIRI, we used a mouse model of partial warm HIRI. We clamped the portal vein for 1 hour and observed the serum alanine transaminase (ALT) levels at different reperfusion times. The serum ALT levels were increased from 0 to 6 hours of reperfusion and then decreased to the normal level up to 72 hours after reperfusion, showing a peak at 6 hours after reperfusion (Figure $1 \mathrm{~A})$. Neutrophils $\left(\mathrm{CD} 45^{+} \mathrm{Ly} 6 \mathrm{G}^{+} \mathrm{CD} 11 \mathrm{~b}^{+}\right)$were detected in the postischemic tissue by flow cytometry, and the gating strategy is shown in Supplemental Figure 1 (supplemental material available online with this article; https://doi.org/10.1172/jci.insight.129736DS1). Similar to the ALT levels, the proportion of hepatic neutrophils was increased and peaked at 6 hours after reperfusion $(27.38 \pm 2.86 \%$ at 6 hours vs. $23.48 \pm 1.93 \%$ at 4 hours, $P=0.035)$ and then rapidly decreased $(27.38 \pm 2.86 \%$ at 6 hours vs. 12.25 $\pm 3.40 \%$ at 8 hours, $P=0.0001$ ), suggesting that ischemia/reperfusion injury peaked 6 hours after reperfusion and was associated with a significantly high proportion of neutrophils (Figure 1, B and C).

Then, we explored OX40 expression in hepatic neutrophils during HIRI. After reperfusion, we obtained hepatic neutrophils from liver MNCs via FACS (purity > 95\%) at different time points and measured the relative mRNA expression of Ox40. Compared with the sham-operated group, Ox40 expression in the neutrophils from the HIRI group was strikingly upregulated at different reperfusion times. However, unexpectedly, Ox40 expression peaked at 1 hour of reperfusion (Figure 1D). To further verify the time course of OX40 expression in neutrophils after HIRI, we also detected the variation in OX40 expression via flow cytometry. In the sham group, the mice exhibited very low levels of OX40 expression in the hepatic neutrophils. Consistent with the mRNA expression, OX40 expression in the hepatic neutrophils reached a maximum at 1 hour of reperfusion and then decreased as the reperfusion time increased. After 6 hours of reperfusion, the expression level of OX40 in the neutrophils from the HIRI group was still higher than that in the sham group (Figure 1, E-G).

Meanwhile, TNF- $\alpha$ and IL-1 $\beta$ synthesized by hepatic neutrophils were also detected. Compared with the sham group, the mRNA expression of Tnfa and Illb all significantly increased after HIRI, while Tnfa peaked at 4 hours and $I l 1 b$ at 2 hours of reperfusion (Figure 1, H and I). The variation tendency of serum TNF- $\alpha$ and IL-1 $\beta$ was also similar to the mRNA (Figure 1, J and K). These results revealed that Ox40 expression in neutrophils was increased after HIRI, which might be associated with proinflammatory cytokines, such as TNF- $\alpha$ and IL-1 $\beta$, directly secreted by neutrophils.

Ox40 knockout ameliorates HIRI. To explore the role of the OX40 molecule in neutrophils in HIRI, we established a HIRI model in WT and $O x 40^{-1-}$ mice. As shown in Figure 2A, lower levels of serum ALT were observed in the $O x 40^{-1-}$ mice at different reperfusion times. This difference was significant after 6 hours of reperfusion (1988.09 $\pm 433.83 \mathrm{IU} / \mathrm{L}$ in WT HIRI vs. $695.40 \pm 158.52 \mathrm{IU} / \mathrm{L}$ in $O x 40^{-/-} \mathrm{HIRI}, P=$ 0.0001) (Figure 2B). Compared with the WT mice, the $O x 40^{-1-}$ mice showed less hepatocellular necrosis, indicating that the Ox40 depletion ameliorated HIRI (Figure 2C).

The flow cytometric analyses showed markedly less liver neutrophilic infiltration in the $O x 40^{-1-}$ mice as determined by the lower proportion of $\mathrm{CD} 45^{+}$cells and absolute numbers (Figure 2, D-F). This finding was consistent with lower serum myeloperoxidase (MPO) level and the lower mRNA level of MPO in the hepatic MNCs from the $O x 40^{-1-}$ mice (Figure 2, $\mathrm{G}$ and $\mathrm{H}$ ). In addition, the $\mathrm{O} x 40^{-1-}$ mice displayed a lower mRNA level of Nox2 (NAPDH oxidase 2) after HIRI compared with the WT mice (Figure 2I). All these data indicated that the absence of $O x 40$ in the mice ameliorated HIRI, followed by a reduction in neutrophilic infiltration and oxidative burst of neutrophils.

To investigate the role of other factors that also influence neutrophilic infiltration, we also detected the proportion of other important immune cells (2, 16-18), damage-associated molecular pattern molecules (DAMPs) (e.g., Hmgb1 or S100b, ref. 7), and chemotaxis signals (19). We found that the proportions of Kupffer cells, monocytes, and $\mathrm{CD} 4^{+} \mathrm{T}$ cells, but not $\mathrm{CD} 8^{+} \mathrm{T}$ cells, were markedly decreased in $O x 40^{-1-}$ mice (Figure 2, J and K). In addition, the mRNA level of $S 100 b$ was significantly decreased, while Hmgb1 had no significant difference between WT and $O x 40^{-1-}$ mice after HIRI (Figure $2 \mathrm{~L}$ ). The chemotaxis signals, including $C c l 2, C c l 3, C c l 5, C c 19$, were all decreased in $O x 40^{-1-}$ mice (Figure $2 \mathrm{M}$ ).

OX40 in neutrophils contributes to HIRI. To directly confirm that OX40 in neutrophils, but not T cells, contributes to the protection against HIRI, adoptive transfer assays were performed in $\mathrm{Rag} / \mathrm{Il}_{2 \mathrm{rg}}{ }^{-/}$mice lacking residual $\mathrm{T}, \mathrm{B}$, and $\mathrm{NK}$ cells. We used anti-Ly6G mAb to deplete the resident neutrophils and performed adoptive transfer of neutrophils from WT or $O x 4 \mathrm{O}^{-/-}$mice into $\mathrm{Rag} 2 / \mathrm{Il}_{2} \mathrm{rg}^{-1-}$ or $\mathrm{O} \times 4 \mathrm{O}^{-1-}$ mice; then, HIRI models were established. 
A

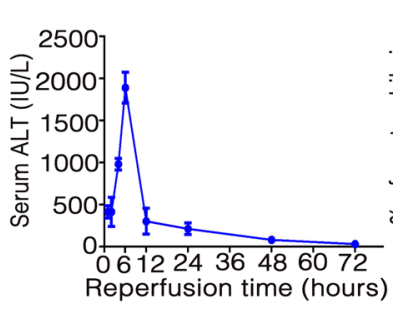

B

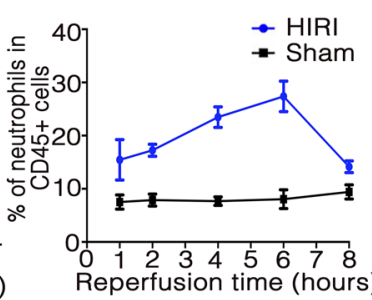

C

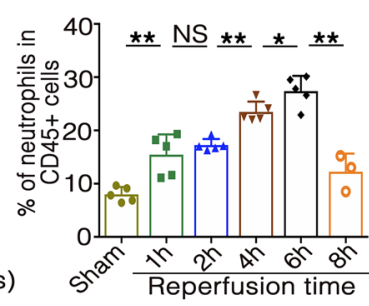

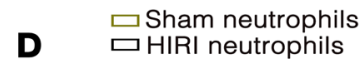

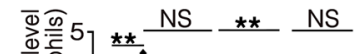

E

Isotype control
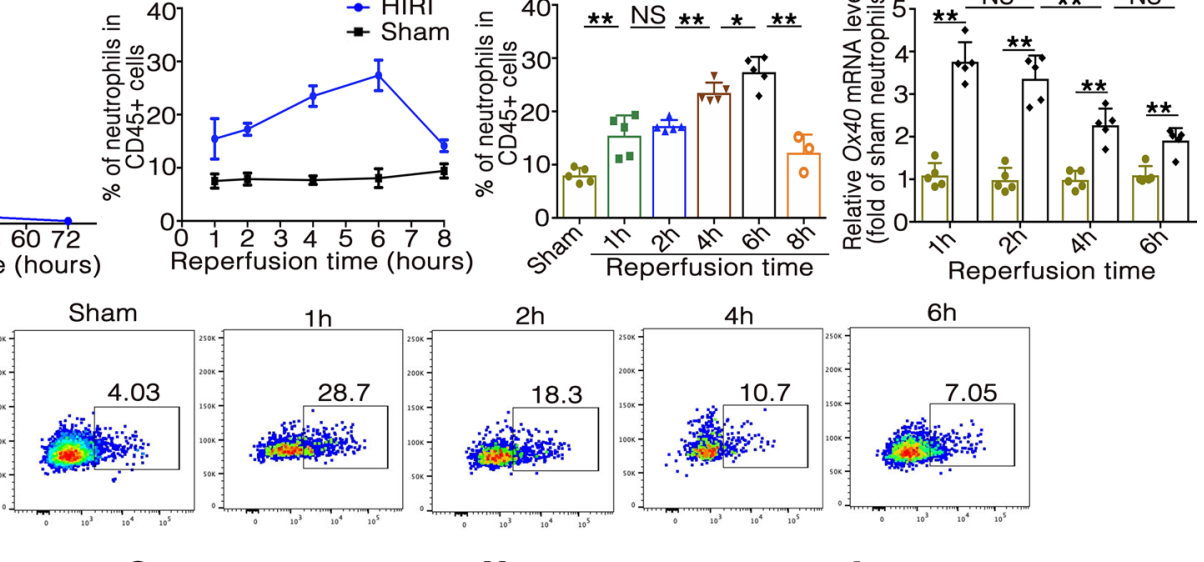

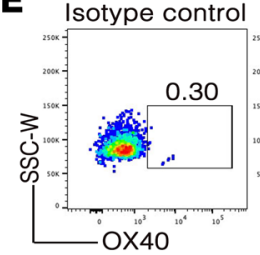

$\mathbf{F}$
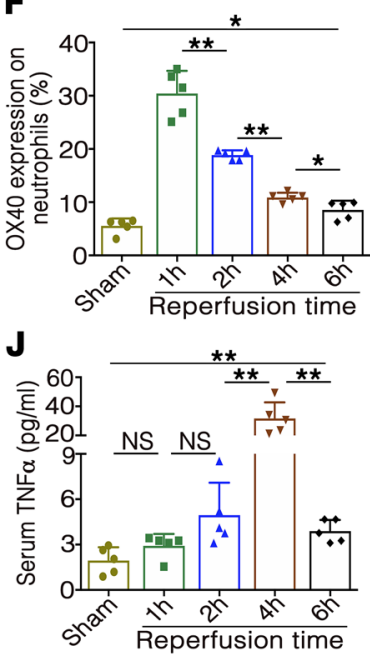

G
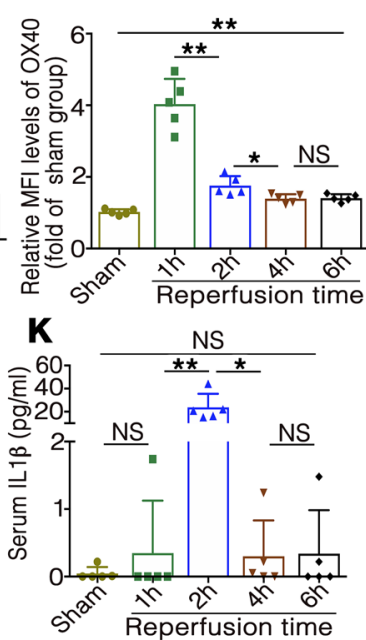

$2 h$

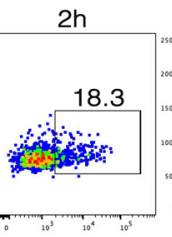

$4 \mathrm{~h}$
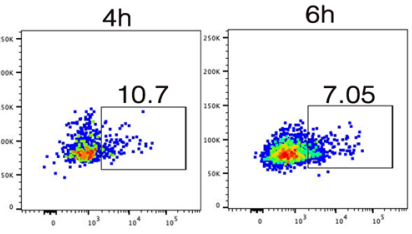

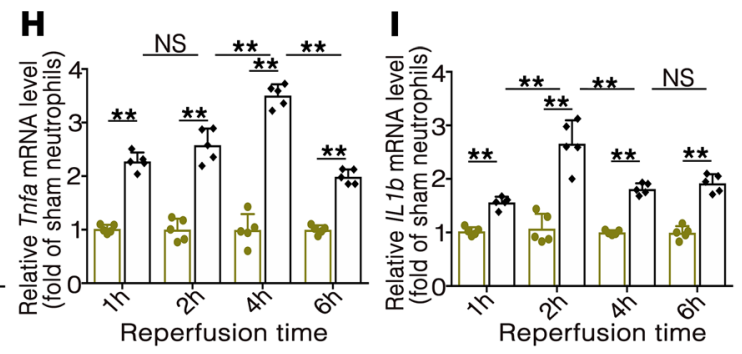

Reperfusion time

Figure 1. HIRI peaked after 6 hours of reperfusion and mediated the upregulation of $\mathbf{0 X 4 0}$ expression in neutrophils. (A) Serum ALT levels following 1 hour of ischemia and different reperfusion times ( 1 hour, 2 hours, 4 hours, 6 hours, 12 hours, 24 hours, 48 hours, and 72 hours after reperfusion) were examined ( $n=$ 3/group). (B) CD45 leukocytes were gated for an analysis of the neutrophil (Ly6G+ and CD11b+ subsets) proportions at different time points (1 hour, 2 hours, 4 hours, 6 hours, and 8 hours after reperfusion), and the neutrophils were characterized by flow cytometry ( $n=3-5 / g r o u p)$. (C) Statistical analysis of neutrophil proportions in $\mathrm{CD} 45^{+}$gates ( $n=3-5$ /group). Neutrophils were isolated from liver mononuclear cells (MNCs) by FACS after different reperfusion times, and relative mRNA expression of $O \times 40$ (D) was measured by real-time PCR $(n=5 /$ group). Representative flow cytometric results $(\mathbf{E})$ and statistical analysis $(\mathbf{F})$ of OX40 expression in neutrophils (CD45+Ly6C + CD11 b subsets) ( $n=5 /$ group). (C) Relative MFI levels of OX40 in neutrophils plotted in each group ( $n=5 /$ group). Neutrophils were isolated from liver MNCs by FACS after different reperfusion times, and relative mRNA expression of Tnfa (H) and IIlb (I) was measured by real-time PCR ( $n=5 /$ group). Serum TNF- $\alpha(\mathrm{J})$ and IL-1 $\beta(\mathbf{K})$ levels $(\mathrm{pg} / \mathrm{mL})$ were measured by mouse inflammation panel $(n=5 /$ group). Data represent mean \pm SD. Significant differences were analyzed using 2-tailed Student's $t$ test and 1-way ANOVA. ${ }^{*} P<0.05 ;{ }^{*} P<0.01$; NS, No significant difference.

The efficiency of the neutrophil depletion in the liver with $400 \mu \mathrm{g}$ anti-Ly6G mAb was examined daily from days 1 to 3 after the antibody injection. As shown in Figure $3 \mathrm{~A}$, almost $80 \%$ of the neutrophils were thoroughly depleted from the liver, and the efficacy was maintained up to 2 days after the $\mathrm{mAb}$ injection. After 3 days, the liver neutrophil frequency started to recover up to $50 \%$ of the initial level before the antibody depletion. We further tested the effects of residual anti-Ly6G mAb in recipient mice on newly adoptive transferred BM neutrophils. The mice were subjected to adoptive transfer and received neutrophils 2 days after the antibody depletion. On the following day ( 3 days after the antibody depletion and 1 day after the neutrophil reconstitution), the recipient mice displayed a frequency of liver neutrophils that was almost comparable to that in the naive mice (Figure 3A). However, in the mice that received the neutrophil reconstitution only 1 day after the antibody depletion, a significantly lower percentage of liver neutrophils was found on the following day ( 2 days after the antibody depletion and 1 day after the neutrophil reconstitution), suggesting that the anti-Ly6G antibody does not have residual effects on newly transferred neutrophils after 2 days. Furthermore, to distinguish self and transferred 
A
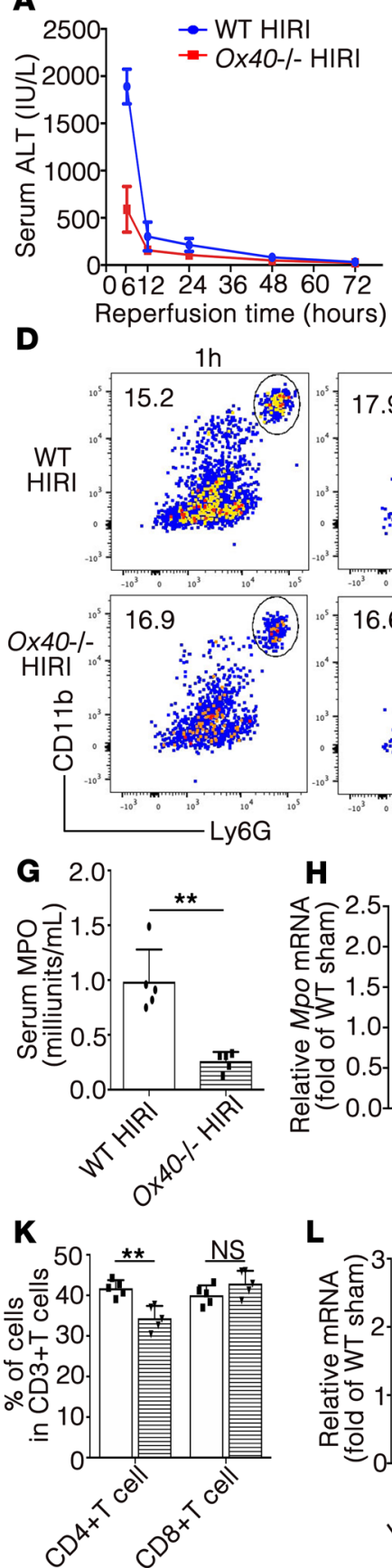

$\mathbf{L}$
B

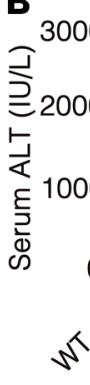

C

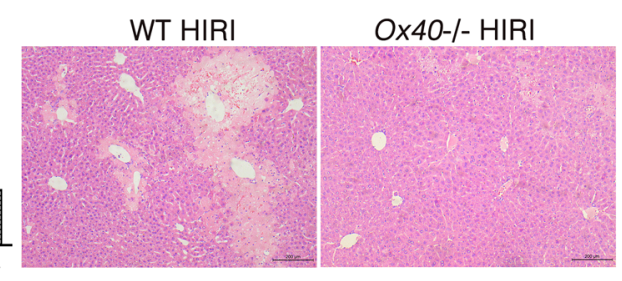

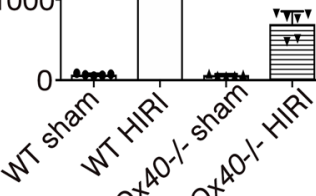

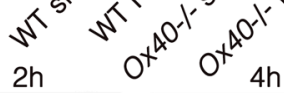

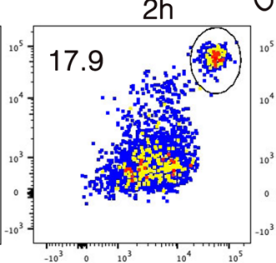

$\sqrt[105]{25.1}$

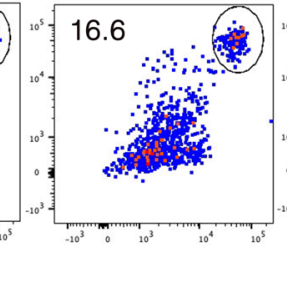

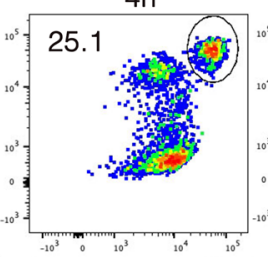

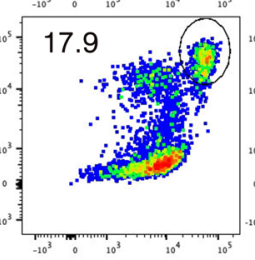

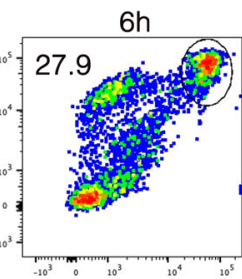

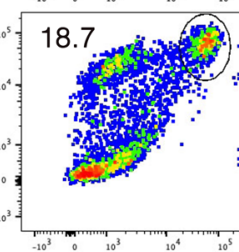

$\mathbf{E} \subseteq \quad 40$

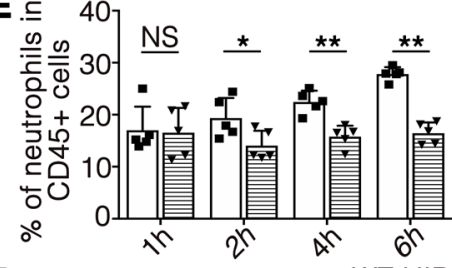

$F$

$\wedge^{n} v^{n} a^{n} \stackrel{6}{H I R I}^{n}$
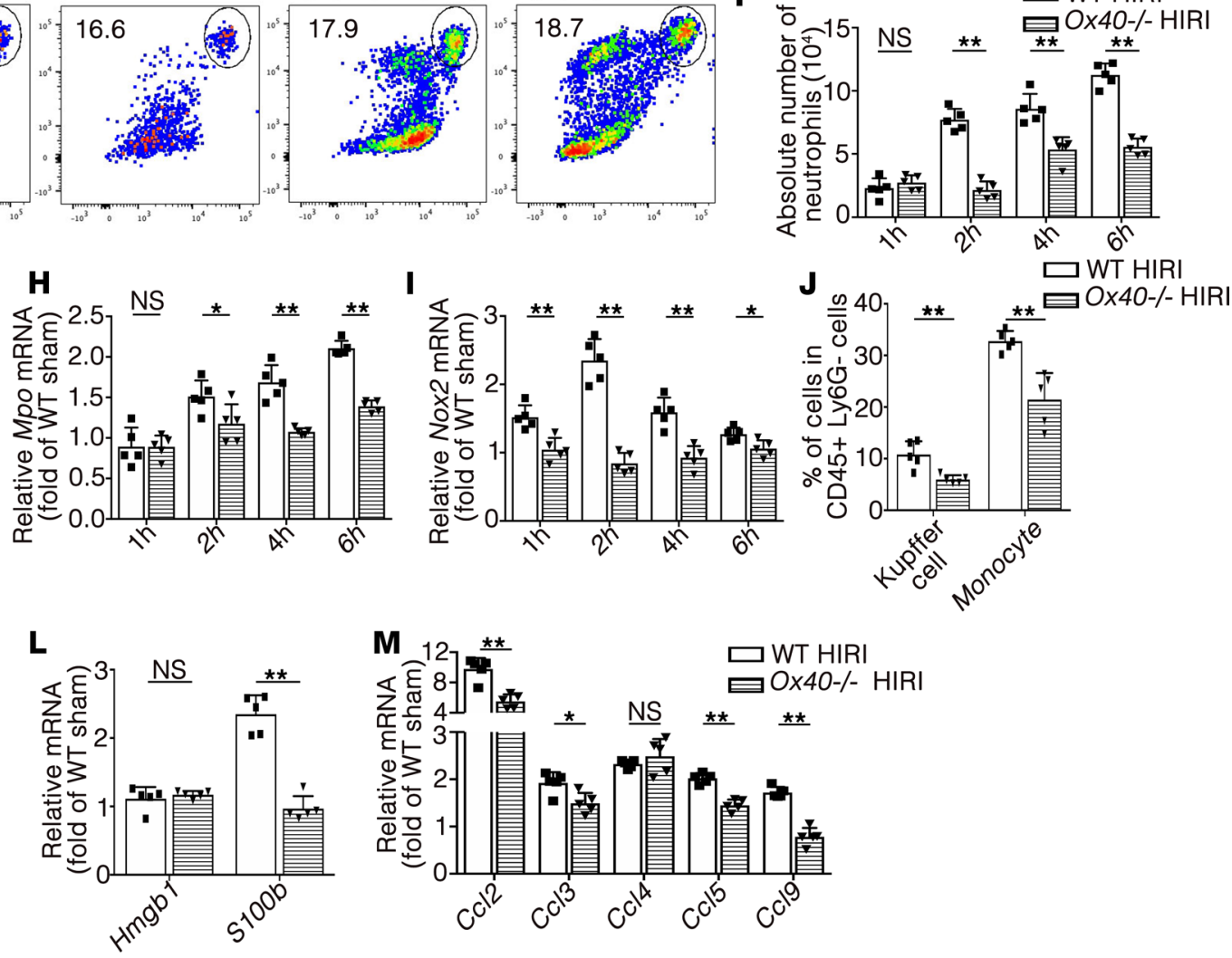

Figure 2. Ox40 knockout reduced HIRI and neutrophil infiltration. (A) Serum ALT curve following 1 hour of hepatic ischemia and different reperfusion times (6 hours, 12 hours, 24 hours, 48 hours, and 72 hours after reperfusion) ( $n=3 / g r o u p$ ). (B) Serum ALT levels following 1 hour of ischemia and 6 hours of reperfusion ( $n=5-6 /$ group). (C) Liver histology in WT and $0 \times 40^{-/-}$mice following 1 hour of ischemia and 6 hours of reperfusion (scale bar: $200 \mu \mathrm{m}$ ). Representative flow cytometric results (D) and statistical analysis (E) of neutrophil proportions in $C^{2} 45^{+}$gates after different reperfusion times $(n=5 / g r o u p)$. $(\mathbf{F})$ Absolute number of neutrophils computed by flow cytometry ( $n=5$ /group). (C) Serum MPO levels following 1 hour of ischemia and 6 hours of reperfusion ( $n=5$ /group). Relative mRNA expression of Mpo (H) and Nox2 (I) in the liver was quantified by real-time PCR ( $n=5 /$ group). The proportions of Kupffer cells and monocytes in CD45+ Ly6G- gates (J) and CD4+ T cells and CD8 ${ }^{+} T$ cells in CD3+ $T$ cells (K) following 1 hour of ischemia and 6 hours of reperfusion ( $n$ $=5$ /group). Relative mRNA expression of $\mathrm{Hmgb1}$ and $5100 b(\mathrm{~L})$ and $C c / 2, C c / 3, C c / 4, C c / 5$, and $C c / 9(M)$ in the liver was quantified by real-time PCR ( $n=5$ ). Data represent mean $\pm S D$. Significant differences were analyzed using Student's $t$ test. ${ }^{*} P<0.05 ;{ }^{*} P<0.01$; NS, nonsignificant.

cells after neutrophil reconstitution, we used C57BL/6 congenic for CD45.1 mice as recipients of neutrophils from WT mice (CD45.1-) after neutrophil depletion. We found that the CD45.1+ self-neutrophils accounted for less than $10 \%$ of whole neutrophils in neutrophil-reconstituted mice on day 3 , while CD $45.1^{+}$monocytes were almost $100 \%$ (Figure 3B). Based on these results, to minimize the influences 


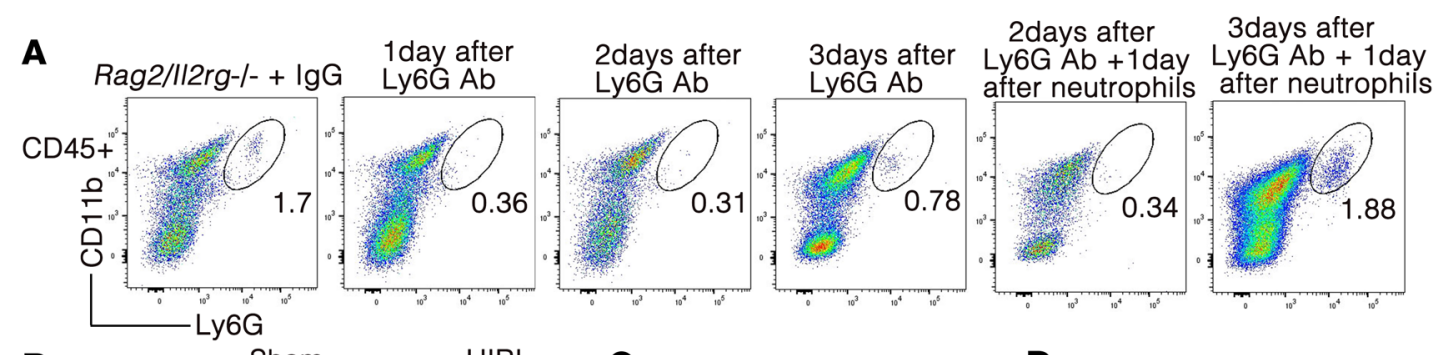

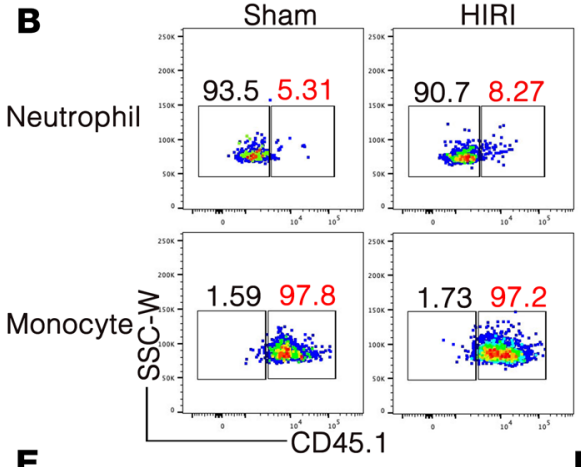

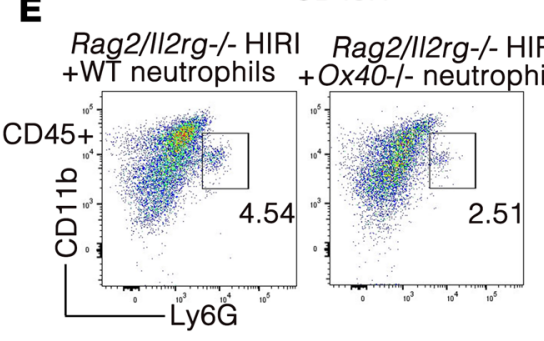

I

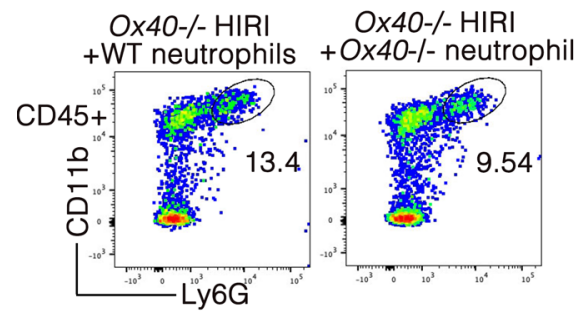

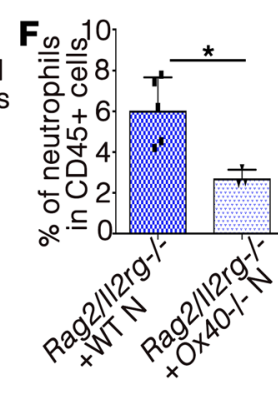

$\mathbf{J}$

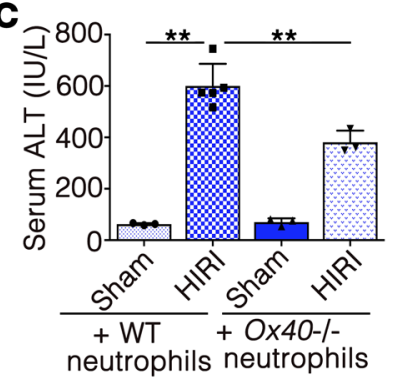

D

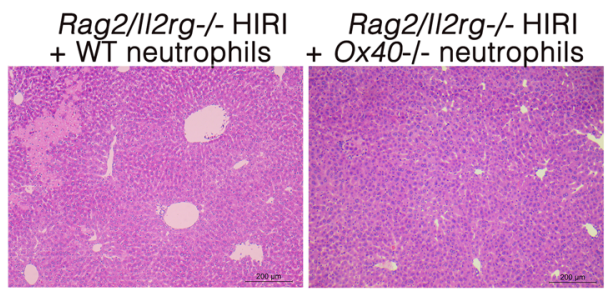

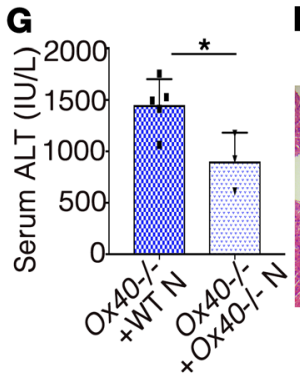

$\mathbf{K}$

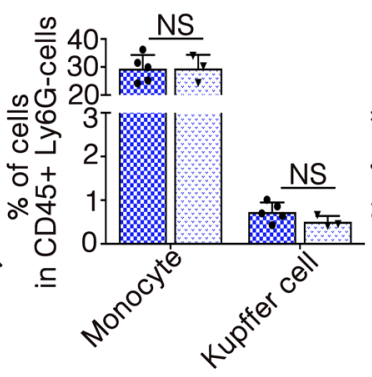
(2)
H Ox40-/-HIRI + WT neutrophils

L $0 \times 40-/-\mathrm{HIRI}+\mathrm{WT}$ neutrophils Ox $0 \times 40-/-\mathrm{HIRI}+\mathrm{O} \times 40-/-$ neutrophils

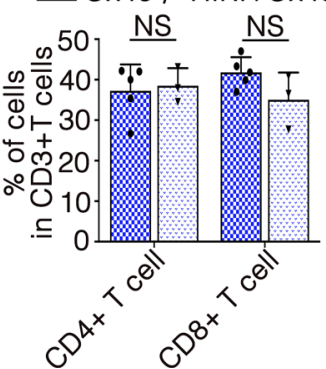

Figure 3. Ox40 deficiency in neutrophils alleviated HIRI and decreased neutrophilic infiltration. (A) Representative flow cytometric results of neutrophil proportions in $\mathrm{CD}_{4} 5^{+}$gates after different treatments with an anti-Ly6C antibody and/or neutrophil adoptive transfer ( $n=3$ independent experiments). (B) C57BL/6 congenic for CD45.1 mice were recipients of adoptive transfer after neutrophil depletion. CD45.1+ neutrophils or monocytes were detected by flow cytometry ( $n=3$ independent experiments). The neutrophil-depleted Rag2/II2 $\mathrm{rg}^{-1-}$ mice were reconstituted with neutrophils from WT or $0 \times 40^{-1-}$ mice. (C) Serum ALT levels following 1 hour of ischemia and 6 hours of reperfusion times ( $n=3-5 /$ group). (D) Representative liver histology after HIRI (scale bar: $200 \mu \mathrm{m})$. Representative flow cytometric results (E) and statistical analyses (F) of proportions of neutrophils in CD45 gates ( $n=3-5 / g r o u p)$. The neutrophil-depleted $0 \times 40^{-1-}$ mice were also reconstituted with neutrophils from WT or Ox40-/- mice. (C) Serum ALT levels following 1 hour of ischemia and 6 hours of reperfusion time ( $n=3-5$ /group). (H) Representative liver histology after HIRI (scale bar: $200 \mu \mathrm{m}$ ). Representative flow cytometric results (I) and statistical analyses (J) of proportions of neutrophils in $\mathrm{CD}_{4} 5^{+}$gates ( $n=3-5$ /group). The proportions of Kupffer cells and monocytes in $\mathrm{CD}^{2} 5^{+}$Ly6C ${ }^{-}$gates (K) and $C D 4^{+} T$ cells and $C D 8^{+} T$ cells in $\mathrm{CD3}^{+} T$ cells $(\mathrm{L})(n=3-5$ /group). Data represent mean \pm SD. Significant differences were analyzed using 2-tailed Student's $t$ test and 1-way ANOVA. ${ }^{*} P<0.05 ;{ }^{*} P<0.01$; NS, nonsignificant.

of the residual antibody on the newly transferred neutrophils and the recovery of self-neutrophils, the mice underwent adoptive transfer with BM neutrophils 2 days after the antibody depletion and were subjected to HIRI on day 3.

As expected, compared with the WT neutrophils, the $O \times 40^{-1-}$ neutrophils protected the liver from HIRI well in Rag2/Il2 $\mathrm{rg}^{-1-}$ mice as shown by apparently low levels of serum ALT (381.00 $\pm 45.43 \mathrm{IU} / \mathrm{L}$ in Rag2/ $\mathrm{Il} 2 \mathrm{rg}^{-1-} \mathrm{HIRI}+\mathrm{Ox}^{-1-}$ neutrophils vs. $600.40 \pm 85.68 \mathrm{IU} / \mathrm{L}$ in $\mathrm{Rag} 2 / \mathrm{Il} 2 \mathrm{rg}^{-/-} \mathrm{HIRI}+\mathrm{WT}$ neutrophils, $\left.P=0.007\right)$ 
and reduced hepatocellular necrosis (Figure 3, C and D). These findings were accompanied by reduced neutrophilic infiltration as shown by flow cytometric analysis $\left(2.71 \pm 0.42 \%\right.$ in $\mathrm{Rag}_{2} / \mathrm{Il} 2 \mathrm{rg}^{-1-} \mathrm{HIRI}+\mathrm{O}^{-1} 40^{-1}$ neutrophils vs. $6.19 \pm 1.63 \%$ in Rag2/Il2 $\mathrm{rg}^{-/-}$HIRI + WT neutrophils, $P=0.023$ ) (Figure 3, E and F).

To verify the role of OX40 in neutrophils and other immune cells in HIRI, we also reconstituted neutrophil-depleted $O \times 40^{-1-}$ mice with WT or $O X 40^{-1-}$ neutrophils. Similarly, $O \times 40^{-1-}$ neutrophils resulted in less liver injury, as shown by decreased ALT levels $\left(901.76 \pm 280.13 \mathrm{IU} / \mathrm{L}\right.$ in $O x 40^{-1-} \mathrm{HIRI}+O \times 40^{-/-}$neutrophils vs. $1451.15 \pm 251.24 \mathrm{IU} / \mathrm{L}$ in $O x 40^{-1-} \mathrm{HIRI}+\mathrm{WT}$ neutrophils, $\left.P=0.028\right)$ and less hepatocellular necrosis (Figure 3, G and H). Decreased neutrophil infiltration (9.89 $\pm 0.79 \%$ in $O x 40^{-1-} \mathrm{HIRI}+O x 40^{-/-}$neutrophils vs. $13.6 \pm 2.24 \%$ in $O x 40^{--}$HIRI + WT neutrophils, $P=0.036$ ) was also detected in $O x 40^{-/-}$neutrophils (Figure 3, I and J). Interestingly, no significant differences of proportions of other cells, including monocytes, Kupffer cells, CD4 ${ }^{+} \mathrm{T}$ cells, or $\mathrm{CD}^{+} \mathrm{T}$ cells (Figure 3, $\mathrm{K}$ and $\mathrm{L}$ ) were found between WT or Ox40-1neutrophil-reconstituted mice, suggesting that $O \times 40$ did affect the function of neutrophils even if without the influence from other immune cells.

OX40 facilitates the inflammatory reaction and increases neutrophil survival. To explore the potential mechanisms of OX40 in neutrophils in HIRI, in vitro stimulation assays were performed. The naive BM neutrophils did not show obvious expression of OX40 by flow cytometry; however, following PMA/ionomycin stimulation, the activated neutrophils exhibited markedly upregulated OX40 expression $(0.61 \pm 0.14 \%$ in WT neutrophils vs. $12.43 \pm 0.74 \%$ in WT neutrophils + PMA, $P=0.0001)$, which was consistent with the real-time PCR results (Figure $4, \mathrm{~A}$ and $\mathrm{B}$ ).

Consistent with the results of the animal experiments, stimulation with PMA/ionomycin also increased the production of proinflammatory cytokines, such as TNF- $\alpha$ and IL-1 $\beta$, and Ox40 deficiency statistically decreased the secretion of these cytokines (Figure 4, C and D). Moreover, the Ox40 deficiency also displayed appreciably decreased mRNA levels of Il17a, Rorgt, and Nfkb. We also noticed that the $O x 40$ deficiency downregulated the expression of the chemokine receptors Ccr 1 and Ccr2 in the neutrophils (Figure 4E). To explore the effect of OX40 on oxidative burst, intracellular ROS production was measured by 2,7-dichlorodihydrofluorescein diacetate (DCFH-DA), and we found an evidently reduced level of ROS production in the $O x 40^{-1-}$ neutrophils (Figure 4F). This finding was also consistent with the decreased mRNA level of $M p o$ and Nox2, which are index measures of neutrophil activation (Figure 4E). These data indicate that OX40 could promote neutrophil proinflammatory cytokine secretion and oxidative burst.

To support the role of OX40 in neutrophil survival, we quantified the mRNA expression of Bcl2 and Bid via real-time PCR and measured annexin V via flow cytometry. Expectedly, the $O x 40$ deficiency increased neutrophil apoptosis compared with that observed in the neutrophils from the WT mice (Figure 4, E and G).

Ox40 knockout inhibits $N F-\kappa B /$ RelA in neutrophils via a Traf1/2/4-related pathway. Previous studies have demonstrated well that Traf and NF-кB are essential for OX40 downstream signaling in T cells (20, 21). To explore the potential mechanisms of OX40 in neutrophils, we examined the mRNA expression of Traf1/2/3/4/5/6. As shown in Figure 5A, treatment with PMA/ionomycin specifically upregulated the mRNA expression of Traf1/2/4, and Ox40 deficiency decreased neutrophil Traf1/2/4 expression. Meanwhile, the flow cytometric analysis displayed that $O x 40$ knockout decreased the phosphorylation levels of protein kinase B (AKT), inhibitor of nuclear factor $\kappa-B$ kinase subunit $\alpha / \beta$ (IKK $\alpha / \beta)$, and

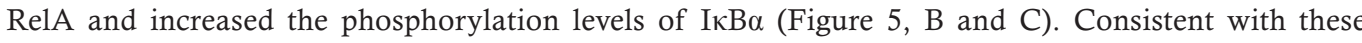
data, Ox40 deficiency significantly depressed the expression of nuclear proteins, including NF- $\kappa \mathrm{B}(\mathrm{p} 50 /$ p105) and RelA, compared with those observed in the neutrophils from the WT mice (Figure 5, D and E). Taken together, these observations suggest that OX40 regulates the function of neutrophils via the Traf1/2/4-associated NF-кB pathway.

OX40L protein and OX40 agonist promote the neutrophil activation and survival. After 1 hour of reperfusion, when OX40 expression on neutrophils reached maximum level, OX40L expression was also significantly upregulated in the liver compared with the sham group (Figure 6A). To explore the function of OX40L/ OX40 interaction on neutrophils, we further tested neutrophils after the stimulation of OX40 on neutrophils by OX40L or OX40 agonist OX86. As expected, administration of OX40L protein promoted neutrophils' ROS generation, and this activation effect was neutralized by the administration of OX40L-blocking antibody. Similarly, OX86 also increased ROS generation of neutrophils, compared with the control rat IgG (Figure 6B). As shown in Figure 6, D and E, we also found that exogenous administration of OX40L and OX86 markedly decreased the proportion of 7-AAD ${ }^{+}$annexin $\mathrm{V}^{+}$and 7-AAD ${ }^{-}$annexin $\mathrm{V}^{+}$cells and increased 
A

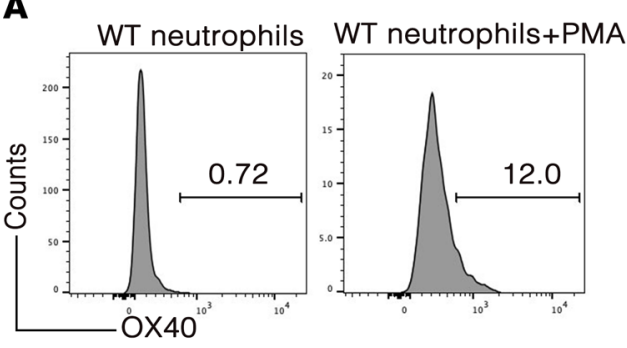

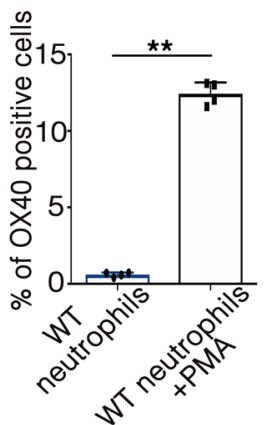

WT neutrophils $0 \times 40-/-$ neutrophils

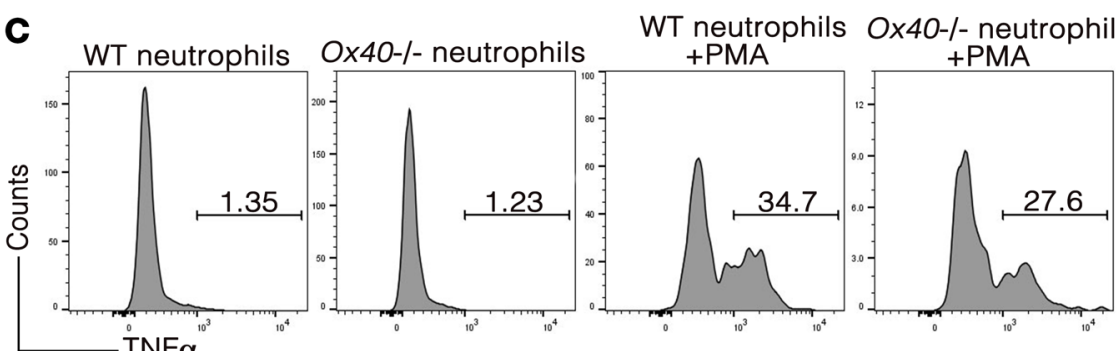

TNF $\alpha$
WT neutrophils Ox40-/- neutrophils

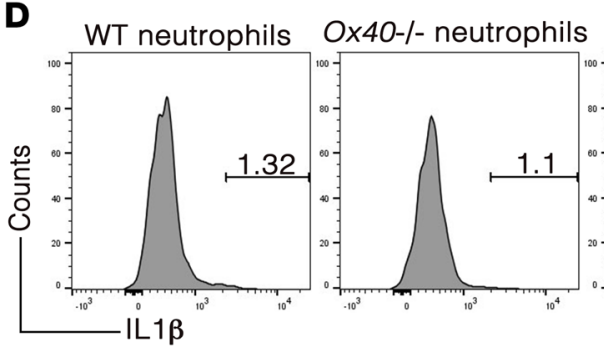
+ PMA

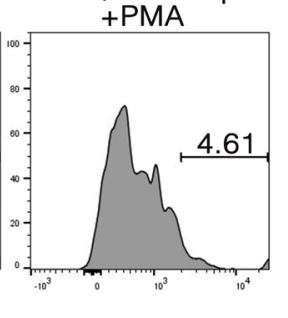

B $\overparen{\frac{0}{\bar{c}}}$
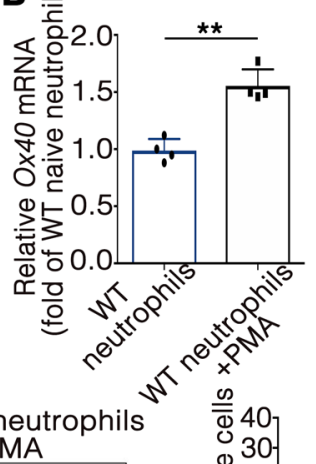
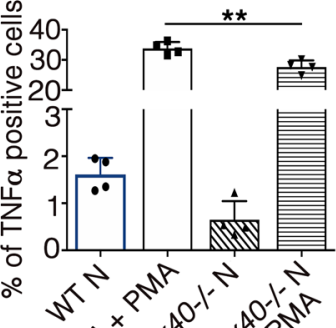

25
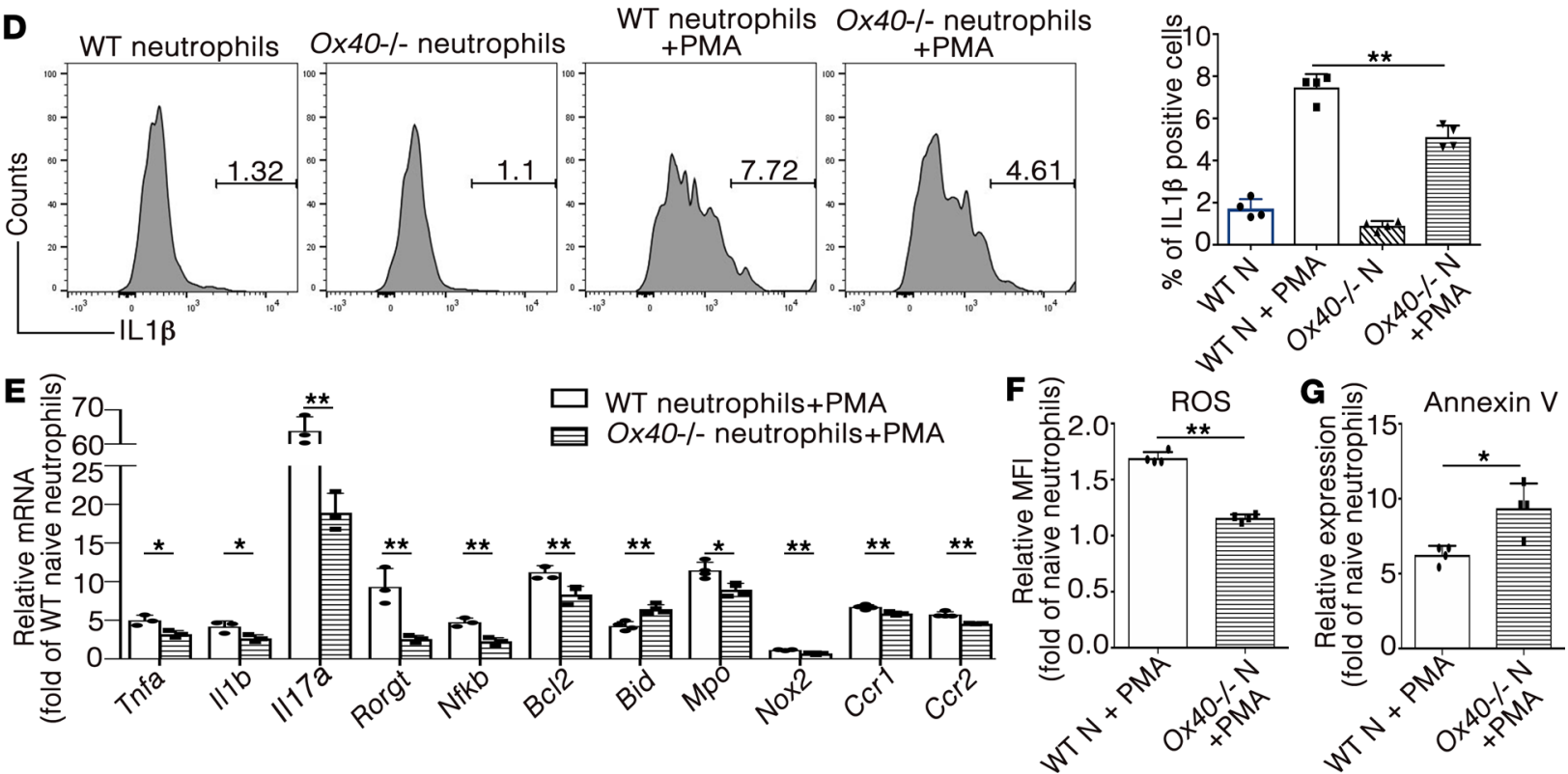

Figure 4. $0 \times 40$ in neutrophils promoted cytokine and ROS production and was involved in neutrophil infiltration and prolonged cell survival.

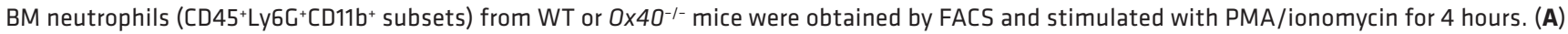
Representative flow cytometric results and statistical analyses of OX40 expression in neutrophils ( $n=4 /$ group). (B) Relative OX4O mRNA expression in neutrophils ( $n=4$ /group). Representative flow cytometric results and statistical analyses of TNF- $\alpha$ (C) and IL-1 $\beta$ (D) produced by neutrophils ( $n=4$ / group). (E) Relative mRNA expression of Tnfa, I/1b, I/17a, Rorgt, Nfkb, Bcl2, Bid, Mpo, Nox2, Ccr1, and Ccr2 in each group ( $n=3-4 / g r o u p)$. (F) Relative MFI levels of ROS measured by flow cytometry ( $n=4$ /group). (G) Relative annexin $\mathrm{V}^{+}$percentage measured by flow cytometry ( $n=4 /$ group). Data represent mean \pm SD. Significant differences were analyzed using Student's $t$ test. ${ }^{*} P<0.05 ;{ }^{*} P<0.01$.

7-AAD-annexin $\mathrm{V}^{-}$cells, while OX40L-blocking antibody completely reversed the antiapoptosis effect of OX40L protein on neutrophils. Similarly, OX40L significantly enhanced neutrophil viability and cytokine production, and the OX40L-blocking antibody neutralized the activation effect of OX40L (Figure 6, C, F, and G). All these data revealed that the activation of OX40 by OX40L and OX86 delivered an indispensable signal to neutrophils, regulating cell survival and oxidative stress reaction.

To strengthen this conclusion, we also observed neutrophilic influx, ROS generation, and cell survival after intraperitoneal injection of $0.25 \mathrm{mg}$ OX86 (22) or rat IgG into $\mathrm{Rag} 2 / \mathrm{Il} 2 \mathrm{rg}^{-1-}$ mice at the beginning of reperfusion. Consistent with the results of experiments in vitro, OX86 injection significantly increased neutrophil infiltration and ROS generation in vivo, while enhancing cell survival (Figure 6, H-J). 


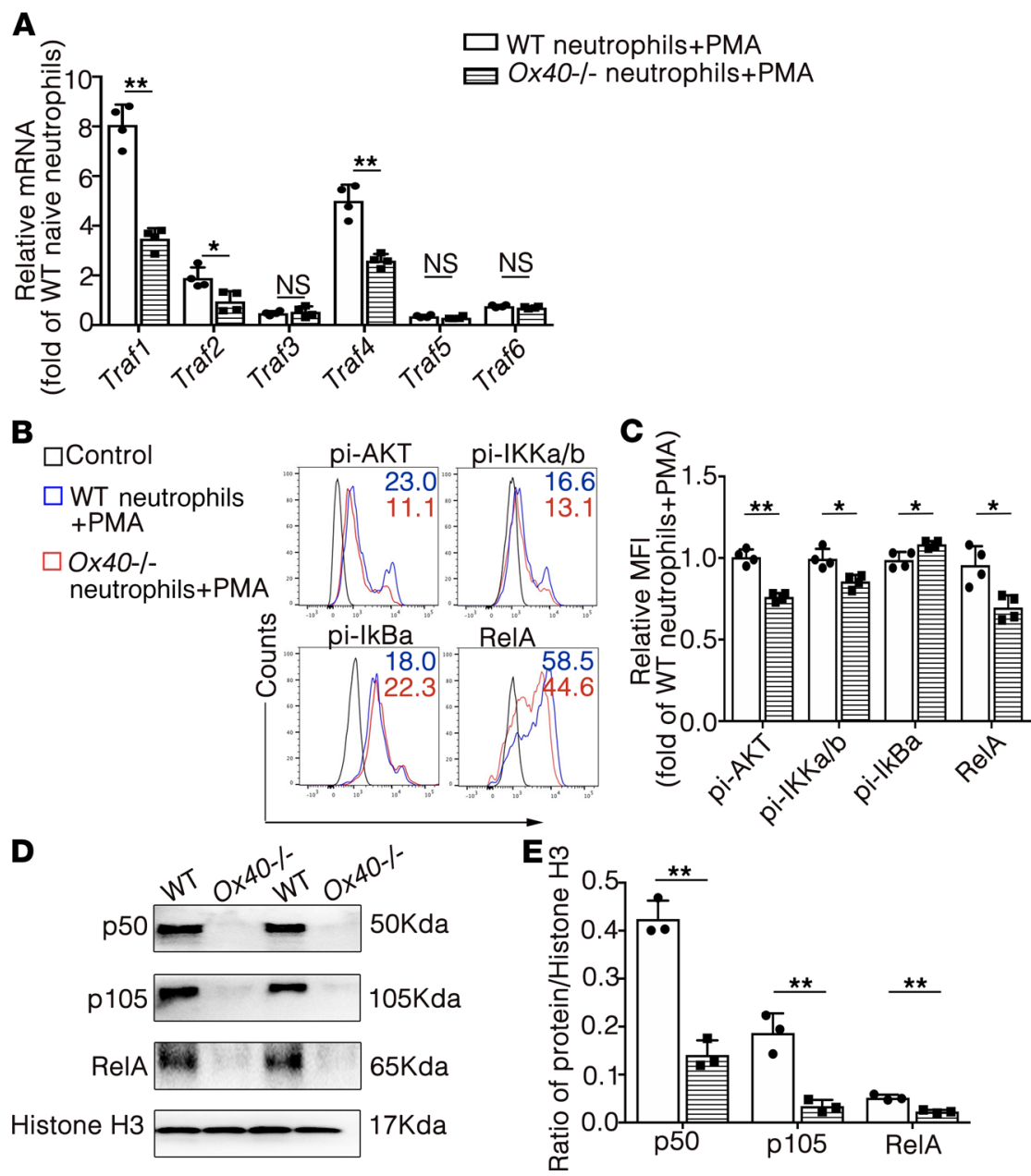

Figure 5. OX40 knockout inhibited NF-кB1/RelA in neutrophils via a Traf1/2/4-related pathway. BM neutrophils (CD45+Ly6C +CD11b+ subsets) from WT or Ox40-1- mice were obtained by FACS and stimulated with PMA/ionomycin for 4 hours. (A) Relative mRNA expression of Traf1, Traf2, Traf3, Traf4, Traf5, and Traf6 was examined in each group $(n=$ 4/group). Representative flow cytometric results (B) and statistical analyses (C) of RelA and phosphorylation of AKT, $I K K \alpha$, and IKB $\alpha$ in WT and $O \times 40^{-1-}$ neutrophils ( $n=4 /$ group). (D) Nuclear proteins were extracted from neutrophils, and the relative protein levels of NF- $\mathrm{KB}$ (p50/p105) and RelA were analyzed by Western blotting. (E) Statistical analyses of Western blotting results ( $n=3$ /group). Data represent mean \pm SD. Significant differences were analyzed using Student's $t$ test. ${ }^{*} P<0.05 ;{ }^{* *} P<0.01$; NS, nonsignificant.

\section{Discussion}

HIRI is a pathophysiological process that further aggravates liver injury after blood flow is restored to the liver. During the early phase of HIRI (0-6 hours), DAMPs released by damaged cells activate Kupffer cells and trigger initial damage via ROS and proinflammatory cytokines, inducing excessive neutrophil influx and a cascade of further inflammatory responses $(7,23,24)$.

In this study, we found that neutrophilic infiltration peaked 6 hours after reperfusion at the time of the maximum level of liver injury. In addition to ROS and protease reported in a previous study (24), neutrophils also generate proinflammatory cytokines, such as TNF- $\alpha$ and IL-1 $\beta$, in hepatic ischemia and reperfusion. More importantly, we revealed that the expression of OX40 in neutrophils was vigorously upregulated in HIRI in a time-dependent manner. This finding extends the previous view that OX40 is mainly a functional $\mathrm{T}$ cell costimulatory molecule that is mainly expressed in activated $\mathrm{CD} 4^{+}$cells and $\mathrm{CD} 8^{+} \mathrm{T}$ cells to regulate $\mathrm{T}$ cell activation, differentiation, cytokine production, and survival $(10,11,25)$. Similar to the time course of its expression in T cells $(26,27)$, the level of OX40 expression in neutrophils varied at different reperfusion times. Our detailed study showed that OX40 expression on neutrophils reached a maximum level 1 hour after reperfusion and then gradually declined as the reperfusion time increased. This peak expression time of the OX40 molecule in neutrophils was earlier than that of the maximum neutrophilic liver infiltration, 
A

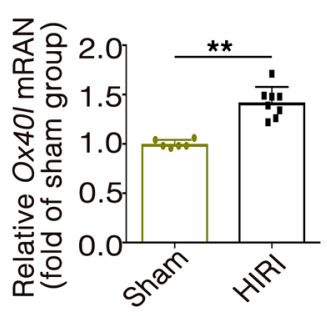

B

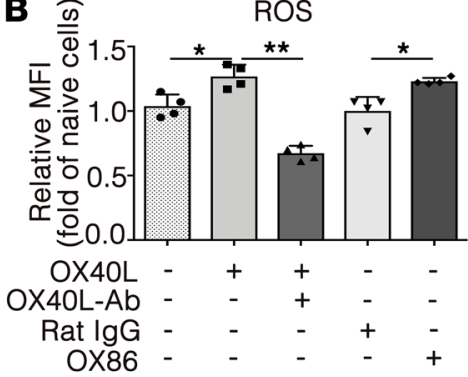

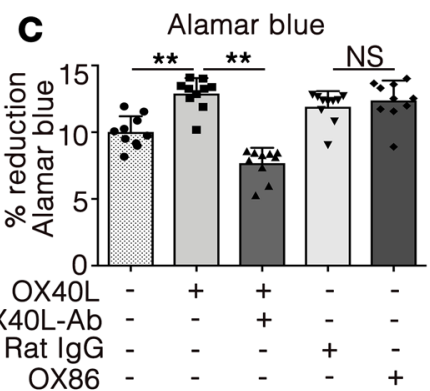

D

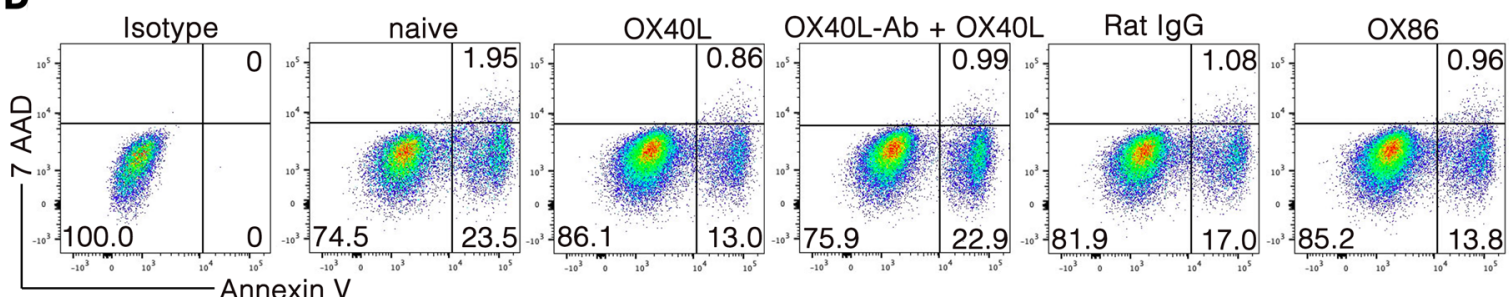

E

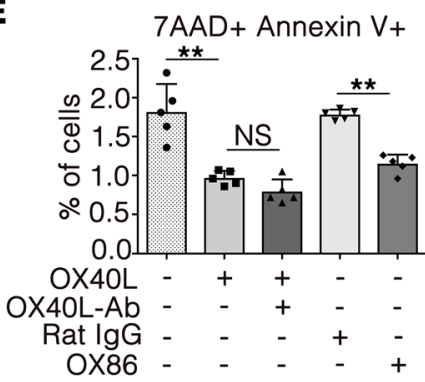

7AAD- Annexin $\mathrm{V}_{+}$

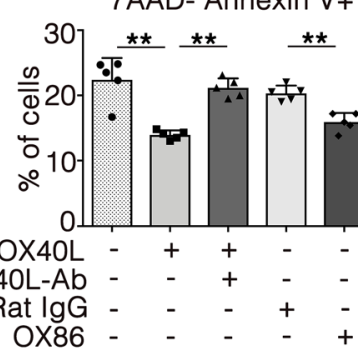

$\mathbf{F}$

\section{G}

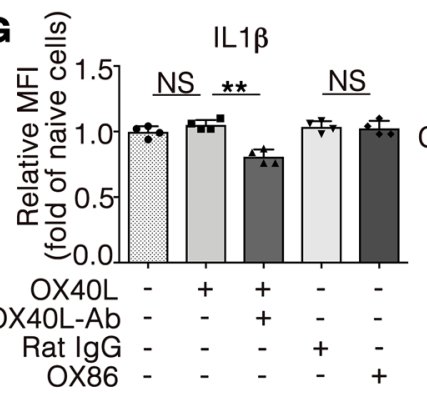

H

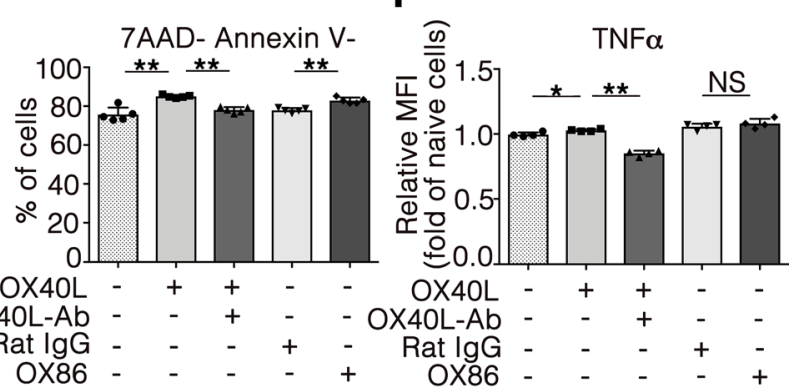

$\mathrm{TNF} \alpha$

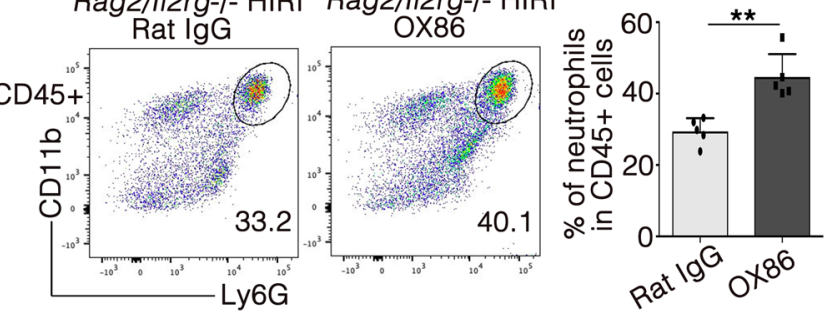

I

I ROS

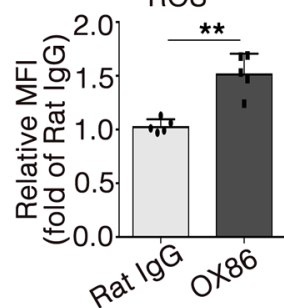

Rag2/II2rg-/- HIRI Rag2/II2rg-/- HIRI

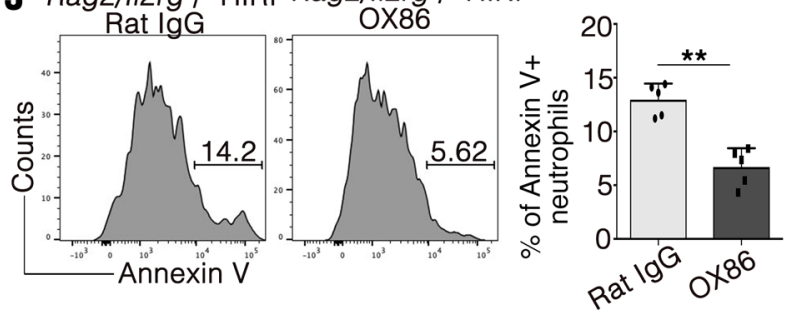

Figure 6. OX40L and OX40 agonist enhanced neutrophil activation and survival. (A) Relative mRNA expression of $O x 40$ lin the liver was measured by real-time PCR after 1 hour of reperfusion ( $n=6$-8/group). BM neutrophils (CD45+Ly6C + CD11b $b^{+}$subsets) from WT mice were obtained by FACS and stimulated with or without $\mathrm{OX} 40 \mathrm{~L}$-blocking antibody $(2 \mu \mathrm{g} / \mathrm{mL})$ for 3 hours followed by $\mathrm{OX} 40 \mathrm{~L}$ protein $(0.5 \mu \mathrm{g} / \mathrm{mL})$ for 6 hours. In other experiments, neutrophils were treated with OX40 agonist mAb OX86 (50 $\mathrm{g} / \mathrm{mL})$ or control rat IgG1 $(50 \mu \mathrm{g} / \mathrm{mL})$ for 6 hours. (B) Relative MFI levels of ROS in neutrophils were measured by flow cytometry ( $n=4$ /group). (C) Alamar Blue assay in each group ( $n=10 /$ group). Representative flow cytometric results (D) and statistical analyses (E) of annexin $\mathrm{V}^{+}, 7$-aminoactinomycin D-positive (7-AAD+) neutrophils measured by flow cytometry ( $n=5 /$ group). Relative MFI levels of TNF- $\alpha$ (F) and IL-1 $\beta$ (C) produced by neutrophils ( $n=4 /$ group). Rag2/II2 $\mathrm{rg}^{-1-}$ mice were intraperitoneally injected with $0.25 \mathrm{mg}$ rat lgG or OX40 agonist mAb (clone OX86, BioXCell) in $0.4 \mathrm{~mL}$ Dulbecco's PBS (DPBS) at the beginning of reperfusion. (H) Representative flow cytometric results and statistical analyses of proportions of neutrophils in $\mathrm{CD}_{4} 5^{+}$gates ( $n=5$ /group). (I) Relative MFI levels of ROS in neutrophils were measured by flow cytometry ( $n=5 /$ group). (J) Representative flow cytometric results and statistical analyses of annexin $V^{+}$neutrophils measured by flow cytometry ( $n=5 /$ group). Data represent mean \pm SD. Significant differences were analyzed using 2 -tailed Student's $t$ test and 1-way ANOVA. ${ }^{*} P<0.05$; ${ }^{* *} P<0.01$; NS, nonsignificant. 
suggesting that OX40 may be involved during the early phase of neutrophil activation, recruitment, and even immunological function. The activation of neutrophils is mainly initiated through ROS and proinflammatory cytokines in HIRI; because neutrophils express OX40, we believe that the OX40/OX40L interaction is another important pathway for the activation of neutrophils by OX40L-expressing antigen-presenting cells in HIRI. This hypothesis needs to be addressed in the future.

In this study, we found that $O x 40$ deficiency in neutrophils remarkably reduced liver injury and neutrophilic infiltration, revealing that $O x 40$ in neutrophils contributes to HIRI. Through an in vitro stimulation assay, we found that naive neutrophils stimulated with PMA/ionomycin exhibited a markedly increased expression of OX40. Il17a and Rorgt were upregulated after the stimulation and dramatically reduced in the $O x 40$-deficient neutrophils. As expected, we also observed that $O x 40$ deficiency resulted in remarkably decreased TNF- $\alpha$ and IL-1 $\beta$ secretion by neutrophils. These findings reveal that OX40 could promote proinflammatory cytokine secretion by neutrophils. Meanwhile, the expression of Nox2 and Mpo was decreased in the Ox40-deficient neutrophils. Because the NOX2/MPO axis is critical for oxidative burst in neutrophils, which is relevant to redundant reactive oxidant generation and tissue damage $(28,29)$, we speculate that OX40 may be involved in oxidant generation and subsequent oxidative stress in neutrophils.

In addition, OX40/OX40L signaling increases cell chemotaxis and mobility by upregulating CXCR5 expression in $\mathrm{CD}^{+} \mathrm{T}$ cells (30) and increasing RANTES (regulated on activation, normal $\mathrm{T}$ cell expressed and secreted)/CCL5 in vascular endothelial cells (31). In neutrophils, CCR1 and CCR2 reportedly play a pivotal role in cell migration to sites of inflammation and promote subsequent inflammatory responses (32-34). We detected the expression of $C c r 1$ and $C c r 2$ and found that neutrophils stimulated with PMA/ ionomycin exhibited an upregulation of these chemotactic factors, whereas the $O x 40$ deficiency slightly decreased this tendency. In addition, this finding could be a potential reason explaining the reduced neutrophilic influx in the $O x 40$-deficient group.

Previous studies have demonstrated well that the activation of OX40 in T cells is crucial for promoting antiapoptotic Bclxl and Bc12 expression and inhibiting cell apoptosis through the NF- $\mathrm{KB}$ pathway $(11,35,36)$. A recent study indicated that OX40 is expressed in human peripheral blood neutrophils and delays cell apoptosis by inhibiting Bid and Bax and maintaining Mcl1 levels, ultimately preventing mitochondrial death (15). We support that OX40 prevents neutrophil apoptosis by showing that $O x 40$ knockout remarkably decreased antiapoptotic $B c l 2$ and increased proapoptotic Bid expression in neutrophils, which were accompanied by the downregulation of $N f k b$. Consistent with these results, $O x 40$ deficiency in the neutrophils increased the number of annexin $\mathrm{V}^{+}$cells. Furthermore, our detailed mechanistic study showed that $O x 40$ knockout significantly inhibited the Traf1/2/4-related NF- $\mathrm{kB1} /$ RelA signaling pathway. In addition, OX40, which targets the intracellular NF- $\mathrm{kB}$ signaling pathway, can promote cytokine production, chemotaxis, and survival (21).

Neutrophils are central players of the innate immune system and constitute a first line of defense against invading pathogens and damaged cells. They exist in a resting state in the blood flow and become primed by DAMPs, including heat shock proteins, high-mobility group box 1 (HMGB1), S100b, purine nucleotides, and uric acid, as well as pathogen-associated molecular pattern molecules, such as bacterial LPS, peptidoglycan, flagellin, and double-stranded RNA $(7,37,38)$. Neutrophils could be also activated by cytokines generated by other immune cells or chemokines $(19,39,40)$. Moreover, in this study, we revealed a potentially new pathway of neutrophil activation via stimulation of cell surface molecular OX40. Both OX40L and OX40 agonist mAb (OX86) effectively inhibited neutrophil apoptosis and promoted ROS generation via strengthening OX40 signaling, which suggests that neutrophils could also be activated by other OX40L-expressing cells, e.g., Kupffer cells, during liver inflammation and HIRI.

Based on our data, we deduce the mechanisms by which OX40 in neutrophils is involved in HIRI. The stimulation of OX40 delivers an indispensable signal to neutrophils; OX40 targets Traf1/2/4-associated $\mathrm{NF}-\mathrm{\kappa B}$ activation, which regulates the biological functions of neutrophils, including the promotion of cytokine production, oxidative burst, cell chemotaxis, and survival, and the number of neutrophils accumulated in the inflammatory liver is increased, exaggerating the cascade of the inflammatory response and leading to further progressive HIRI.

In conclusion, our study provides a new understanding of OX40, which is expressed not only in adaptive immune cells but also in innate immune cells, i.e., neutrophils, contributing to the activation and survival of neutrophils. These findings provide a novel potential therapeutic target for the prevention of HIRI during liver transplantation or hepatic surgery. 


\section{Methods}

Mice. Six- to eight-week-old male WT C57BL/6, C57BL/6 congenic for CD45.1, and C57BL/6 OX40-KO $\left(\mathrm{O} \times 40^{-/}\right)$mice were purchased from the Jackson Laboratory (Bar Harbor, Maine, USA). The B6.Rag2/ $\mathrm{Il}_{2 \mathrm{rg}^{-/-}}$mice were purchased from Taconic Biosciences (Germantown, New York, USA). The mice were maintained in a pathogen-free environment at the animal facilities of Beijing Friendship Hospital.

Reagents and antibodies. The fluorochrome-conjugated antibodies against mouse CD45 (clone 30-F11), CD11b (clone M1/70), F4/80 (clone BM8), Ly6G (clone 1A8-Ly6g), CD3 (clone 145-2C11), CD4 (clone GK1.5), CD8 (clone 53-6.7), OX40 (CD134) (clone OX-86), and TNF- $\alpha$ (clone MP6-XT22); biotin antimouse IL-1 $\beta$ (clone B122); cell activation cocktail (423304); and LEGENDplex mouse inflammation panel (740150) were obtained from BioLegend, and anti-mouse phospho-AKT1 (Ser473) (17-9715-42) and streptavidin-APC (17-4317-82) were purchased from eBioscience. Anti-mouse IкB $\alpha$ (phospho-S36) (ab133462), NF-кB (p105/p50) (ab32360), RelA (p65) (ab32536), and histone H3 (ab18521) and donkey anti-rabbit IgG (ab150075) were obtained from Abcam. In addition, anti-mouse phospho-IKKa (Ser176/180) (16A6) was purchased from Cell Signaling Technology. The PE Annexin V Apoptosis Detection Kit (559763) was purchased from BD Pharmingen. Recombinant mouse OX40L protein (1236OX) and neutralizing antibodies against mouse OX40L (MAB1236) were obtained from R\&D Systems. Rat IgG (I4131) and TRI reagent (93289) were purchased from MilliporeSigma. Anti-mouse Ly6G mAb (clone 1A8) and OX40 agonist mAb (clone OX86) were purchased from BioXCell.

Mouse HIRI model. The model of partial warm HIRI was induced as previously described (41). Briefly, fasted mice were anesthetized, and a middle incision was performed to expose the liver and portal vein. An atraumatic clip was carefully placed across the portal vein and associated structures to interrupt the blood supply to the median and left lateral lobes. The mice were placed on a heating pad to maintain body temperature at $37^{\circ} \mathrm{C}$. After 60 minutes of ischemia, the clip was carefully removed, and hepatic reperfusion was initiated. After specified times of reperfusion, blood and the ischemic hepatic lobes were collected from the anesthetized mice. The sham-operated mice underwent the same procedure without clamping.

Measurement of ALT levels. The blood samples were obtained after specified times of reperfusion, and the serum was collected by centrifugation at $850 \mathrm{~g}$ for 10 minutes. The serum ALT levels were measured by using an Alanine Aminotransferase Assay Kit (C009-2, Nanjing Jiancheng Bioengineering Institute) according to the manufacturer's instructions.

Measurement of MPO levels. The serum MPO levels were measured by an MPO colorimetric activity assay kit (MAK068, MilliporeSigma). After incubating serum with the assay according to the technical bulletin, samples and standards were measured at the absorbance of $412 \mathrm{~nm}$, and then we calculated the MPO activity using the equation: MPO activity $=($ nmol of 5 -thio-2-nitrobenzoic acid consumed $\times$ sample dilution factor) / (reaction time $\times \mathrm{mL}$ of sample).

Isolation of hepatic MNCs. The isolation of mouse liver MNCs by enzymatic digestion in situ was performed as previously described (42). Under deep anesthesia, the mice were perfused with $30 \mathrm{~mL}$ normal saline via the left cardiac apex. The ischemic lobes were cut into small pieces and digested in $0.01 \%$ type IV collagenase (C5138-1G, MilliporeSigma) for 30 minutes at $37^{\circ} \mathrm{C}$. Then, the mixture was dissociated using a gentle-MACS Dissociator (130-093-235, Miltenyi Biotec). The cell suspension was filtered through a 70- $\mu \mathrm{m}$ nylon cell strainer and centrifuged at $50 \mathrm{~g}$ for 5 minutes; then, the supernatant was centrifuged at $500 \mathrm{~g}$ for 5 minutes. The hepatic MNCs were collected from the cell pellet and resuspended with DPBS.

Purification of neutrophils from the liver or bone marrow. The hepatic MNCs were collected via enzymatic digestion as described above. The BM cells were harvested from the WT or $O x 40^{-1-}$ mice, and the RBCs were removed using erythrocyte lysis buffer (79217, QIAGEN). Then, the neutrophils $\left(\mathrm{CD} 45^{+} \mathrm{Ly}_{6 \mathrm{G}}{ }^{+} \mathrm{CD} 11 \mathrm{~b}^{+}\right.$subsets) from the liver or BM were sorted following a mixed lymphocyte reaction using a FACSAria II cell sorter (BD Biosciences).

Neutrophil depletion in vivo and adoptive transfer model. For the depletion of the neutrophils in vivo, $\mathrm{Rag} 2 / \mathrm{Il}_{2 \mathrm{rg}^{-/-}}, \mathrm{O} \times 4 \mathrm{O}^{-/-}$, or C57BL/ 6 congenic for CD45.1 mice were intraperitoneally injected with $0.4 \mathrm{mg}$ rat anti-mouse Ly6G mAb (clone 1A8, BioXCell) in $0.3 \mathrm{~mL}$ DPBS 48 hours before the adoptive transfer of neutrophils from WT or $O x 40^{-1-}$ mice. The control mice were injected with an equivalent amount of rat IgG. The efficacy of the neutrophil depletion was confirmed by flow cytometry.

Two days after the depletion of the neutrophils in vivo, the Rag2/Il2 $\mathrm{rg}^{-/-}, \mathrm{O} \times 40^{-1-}$, or C57BL/6 congenic for CD45.1 mice were subjected to the adoptive transfer of $4 \times 10^{6} \mathrm{WT}$ or $O x 40^{-/-}$neutrophils by a tail vein injection. Twenty-four hours after the cell transfer, the recipient mice underwent ischemia and reperfusion surgery. 
Measurement of ROS production. The intracellular levels of ROS production were measured using an ROS assay kit (E004, Nanjing Jiancheng Bioengineering Institute) according to the manufacturer's instructions. The cells were seeded in 96-well plates $\left(2 \times 10^{5} /\right.$ well) with or without PMA (P8139-1MG, MilliporeSigma)/ionomycin (56092-82-1, Tocris), and $10 \mu \mathrm{M}$ of DCFH-DA (included in the ROS assay kit) was added. After incubation at $37^{\circ} \mathrm{C}$ for 30 minutes, the cells were washed with DPBS 3 times, and intracellular DCF fluorescence was detected by flow cytometry in the FITC channel.

Alamar Blue cell viability assay. After different treatment, neutrophils were seeded in 96-well plates $\left(1 \times 10^{5}\right.$ /well) with or without $10 \mu \mathrm{L}$ of Alamar Blue cell viability reagent (DAL1025, Thermo Fisher Scientific). After incubation at $37^{\circ} \mathrm{C}$ for 4 hours, plates were measured at the absorbance of $570 \mathrm{~nm}$ and $600 \mathrm{~nm}$. Then the percentage reduction of Alamar Blue was calculated.

Stimulation assay in vitro. The BM neutrophils from the WT or $O X 40^{-1-}$ mice were suspended in complete medium (RPMI 1640, 10-040-CVR, Corning, supplemented with $100 \mathrm{U} / \mathrm{mL}$ penicillin, $100 \mu \mathrm{g} / \mathrm{mL}$ streptomycin, $10 \% \mathrm{FBS}$, and $2 \mathrm{mM}$ glutamine) and seeded in 6 -well culture plates $\left(3 \times 10^{6} /\right.$ well $)$ with or without PMA/ionomycin at $37^{\circ} \mathrm{C}$ for 4 hours. The BM neutrophils from WT mice were also stimulated with or without OX40L-blocking antibody $(2 \mu \mathrm{g} / \mathrm{mL})$ for 3 hours followed by OX40L protein $(0.5 \mu \mathrm{g} /$ $\mathrm{mL}$ ) for 6 hours. In other experiments, the BM neutrophils were treated with OX40 agonist mAb OX86 $(50 \mu \mathrm{g} / \mathrm{mL})$ or control rat IgG1 $(50 \mu \mathrm{g} / \mathrm{mL})$ for 6 hours. The cells were harvested for real-time PCR (RT-PCR) and flow cytometric analyses.

OX86 stimulation in vivo. The Rag2/Il2 $\mathrm{rg}^{-1-}$ mice were intraperitoneally injected with $0.25 \mathrm{mg}$ rat $\mathrm{IgG}$ or OX40 agonist mAb (clone OX86, BioXCell) in $0.4 \mathrm{~mL}$ DPBS at the beginning of reperfusion.

Flow cytometric analysis. Flow cytometry was performed with a FACSAria II flow cytometer (BD Biosciences), and the data were analyzed with FlowJo software (Tree Star Inc.).

RT-PCR. The total RNA was extracted from the liver tissue or cells using TRI reagent or an Eastep Super Total RNA Extraction Kit (LS1040, Shanghai Promega) and reverse-transcribed to cDNA using a PrimeScript RT Reagent Kit (RR037A, TaKaRa). The mRNA levels of the target genes were quantified by RT-PCR using an ABI 7500 Sequence Detection System (Applied Biosystems). The data were normalized to Gapdh gene expression and quantified using the $2-\Delta \Delta \mathrm{Ct}$ method. The primer sequences of the target genes are shown in Supplemental Table 1.

Western blot analysis. The WT or OX40-KO neutrophils were prepared by a cell sorter (FACSAria II, BD Biosciences) and collected after the PMA stimulation. The nuclear proteins were extracted from the WT or OX40-KO neutrophils by nuclear and cytoplasmic extraction reagents (78835, Thermo Fisher Scientific). Equal amounts of proteins were subjected to $12 \%$ SDS-PAGE and transferred to

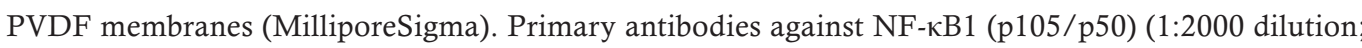
ab32360, Abcam), RelA (1:2000 dilution; ab32536, Abcam), and histone H3 (1:4000 dilution; ab18521, Abcam) and secondary antibodies conjugated to HRP were used (ZB-2301, Zhongshan Golden Bridge Biotechnology Co.). In addition, the immune complexes were detected using chemiluminescence.

Statistics. The significant differences were analyzed using 2-tailed Student's $t$ test and 1-way ANOVA. All statistical analyses were performed using SPSS software (SPSS Inc.). The data were expressed as the mean \pm SD. $P$ values less than 0.05 were considered statistically significant.

Study approval. All animal protocols were approved by the Institutional Animal Care and Ethics Committee of Beijing Friendship Hospital.

\section{Author contributions}

All listed authors participated meaningfully in the study, and they have seen and approved the submission of this manuscript. $\mathrm{HJ}$ and $\mathrm{CZ}$ participated in performing the research, analyzing the data, and initiating the original draft of the article. CS, XZ, DT, WS, YT, KL, and GS participated in performing the research and collecting the data. DZ and HX established the hypotheses, supervised the studies, analyzed the data, and cowrote the manuscript.

\section{Acknowledgments}

This study was supported by grants from the National Natural Science Foundation of China (81570510 and 81870399). 
Address correspondence to: Dong Zhang or Hufeng Xu, Number 95 Yong-an Road, Xi-cheng District, Beijing 100050, China. Phone: 861063139309; Email: zhangd@ccmu.edu.cn (DZ). Phone: 861063138571; Email: xu_hufeng@163.com (HX).

1. Robertson FP, Fuller BJ, Davidson BR. An evaluation of ischaemic preconditioning as a method of reducing ischaemia reperfusion injury in liver surgery and transplantation. J Clin Med. 2017;6(7):E69.

2. Heymann F, Tacke F. Immunology in the liver--from homeostasis to disease. Nat Rev Gastroenterol Hepatol. 2016;13(2):88-110.

3. Zhai Y, Petrowsky H, Hong JC, Busuttil RW, Kupiec-Weglinski JW. Ischaemia-reperfusion injury in liver transplantation--from bench to bedside. Nat Rev Gastroenterol Hepatol. 2013;10(2):79-89.

4. Honda M, Takeichi T, Asonuma K, Tanaka K, Kusunoki M, Inomata Y. Intravital imaging of neutrophil recruitment in hepatic ischemia-reperfusion injury in mice. Transplantation. 2013;95(4):551-558.

5. Adam AN. Some mechanisms of the protective effect of ischemic preconditioning on rat liver ischemia-reperfusion injury. Int $J$ Gen Med. 2014;7:483-489.

6. Quesnelle KM, Bystrom PV, Toledo-Pereyra LH. Molecular responses to ischemia and reperfusion in the liver. Arch Toxicol. 2015;89(5):651-657.

7. Oliveira THC, Marques PE, Proost P, Teixeira MMM. Neutrophils: a cornerstone of liver ischemia and reperfusion injury. Lab Invest. 2018;98(1):51-62.

8. Thomas CJ, Schroder K. Pattern recognition receptor function in neutrophils. Trends Immunol. 2013;34(7):317-328.

9. Tan Z, et al. RORyt+IL-17+ neutrophils play a critical role in hepatic ischemia-reperfusion injury. J Mol Cell Biol. 2013;5(2):143-146.

10. Webb GJ, Hirschfield GM, Lane PJ. OX40, OX40L and autoimmunity: a comprehensive review. Clin Rev Allergy Immunol. 2016;50(3):312-332.

11. Willoughby J, Griffiths J, Tews I, Cragg MS. OX40: structure and function - what questions remain? Mol Immunol. 2017;83:13-22.

12. Publicover J, et al. An OX40/OX40L interaction directs successful immunity to hepatitis B virus. Sci Transl Med. 2018;10(433):eaah5766.

13. Xie K, et al. OX40 expression in hepatocellular carcinoma is associated with a distinct immune microenvironment, specific mutation signature, and poor prognosis. Oncoimmunology. 2018;7(4):e1404214.

14. Lan P, et al. TNF superfamily receptor OX40 triggers invariant NKT cell pyroptosis and liver injury. J Clin Invest. 2017;127(6):2222-2234.

15. Baumann R, Yousefi S, Simon D, Russmann S, Mueller C, Simon HU. Functional expression of CD134 by neutrophils. Eur J Immunol. 2004;34(8):2268-2275.

16. Bilzer M, Roggel F, Gerbes AL. Role of Kupffer cells in host defense and liver disease. Liver Int. 2006;26(10):1175-1186.

17. Song P, et al. Hepatic recruitment of CD11b+Ly6C+ inflammatory monocytes promotes hepatic ischemia/reperfusion injury. Int J Mol Med. 2018;41(2):935-945

18. Caldwell CC, Okaya T, Martignoni A, Husted T, Schuster R, Lentsch AB. Divergent functions of CD4+ T lymphocytes in acute liver inflammation and injury after ischemia-reperfusion. Am J Physiol Gastrointest Liver Physiol. 2005;289(5):G969-G976.

19. McDonald B, Kubes P. Cellular and molecular choreography of neutrophil recruitment to sites of sterile inflammation. J Mol Med. 2011;89(11):1079-1088.

20. Kawamata S, Hori T, Imura A, Takaori-Kondo A, Uchiyama T. Activation of OX40 signal transduction pathways leads to tumor necrosis factor receptor-associated factor (TRAF) 2- and TRAF5-mediated NF-kappaB activation. J Biol Chem. 1998;273(10):5808-5814.

21. Croft M. Control of immunity by the TNFR-related molecule OX40 (CD134). Annu Rev Immunol. 2010;28:57-78.

22. Chonan M, et al. CD40/CD40L expression correlates with the survival of patients with glioblastomas and an augmentation in CD40 signaling enhances the efficacy of vaccinations against glioma models. Neuro-oncology. 2015;17(11):1453-1462.

23. Eggenhofer E, et al. Unconventional ROR $\gamma \mathrm{t}+\mathrm{T}$ cells drive hepatic ischemia reperfusion injury. J Immunol. 2013;191(1):480-487.

24. Xu R, Huang H, Zhang Z, Wang FS. The role of neutrophils in the development of liver diseases. Cell Mol Immunol. 2014;11(3):224-231.

25. Ishii N, Takahashi T, Soroosh P, Sugamura K. OX40-OX40 ligand interaction in T-cell-mediated immunity and immunopathology. Adv Immunol. 2010;105:63-98.

26. Sadler R, et al. Establishment of a healthy human range for the whole blood "OX40" assay for the detection of antigen-specific CD4+ T cells by flow cytometry. Cytometry B Clin Cytom. 2014;86(5):350-361.

27. Evans DE, Prell RA, Thalhofer CJ, Hurwitz AA, Weinberg AD. Engagement of OX40 enhances antigen-specific CD4(+) T cell mobilization/memory development and humoral immunity: comparison of alphaOX-40 with alphaCTLA-4. J Immunol. 2001;167(12):6804-6811.

28. Singel KL, Segal BH. NOX2-dependent regulation of inflammation. Clin Sci. 2016;130(7):479-490

29. Strzepa A, Pritchard KA, Dittel BN. Myeloperoxidase: a new player in autoimmunity. Cell Immunol. 2017;317:1-8.

30. Walker LS, et al. Compromised OX40 function in CD28-deficient mice is linked with failure to develop CXC chemokine receptor 5-positive CD4 cells and germinal centers. J Exp Med. 1999;190(8):1115-1122.

31. Kotani A, Hori T, Matsumura Y, Uchiyama T. Signaling of gp34 (OX40 ligand) induces vascular endothelial cells to produce a CC chemokine RANTES/CCL5. Immunol Lett. 2002;84(1):1-7.

32. Chou RC, et al. Lipid-cytokine-chemokine cascade drives neutrophil recruitment in a murine model of inflammatory arthritis. Immunity. 2010;33(2):266-278.

33. Lewis ND, et al. CCR1 plays a critical role in modulating pain through hematopoietic and non-hematopoietic cells. PLoS ONE. 2014;9(8):e105883.

34. Zhang J, et al. CCL2-CCR2 signaling promotes hepatic ischemia/reperfusion injury. J Surg Res. 2016;202(2):352-362.

35. Rogers PR, Song J, Gramaglia I, Killeen N, Croft M. OX40 promotes Bcl-xL and Bcl-2 expression and is essential for long-term 
survival of CD4 T cells. Immunity. 2001;15(3):445-455.

36. Song J, So T, Croft M. Activation of NF-kappaB1 by OX40 contributes to antigen-driven T cell expansion and survival. J Immunol. 2008;180(11):7240-7248.

37. Kolaczkowska E, Kubes P. Neutrophil recruitment and function in health and inflammation. Nat Rev Immunol. 2013;13(3):159-175.

38. Tan SY, Weninger W. Neutrophil migration in inflammation: intercellular signal relay and crosstalk. Curr Opin Immunol. 2017;44:34-42.

39. Tohme S, et al. Computational analysis supports IL-17A as a central driver of neutrophil extracellular trap-mediated injury in liver ischemia reperfusion. J Immunol. 2019;202(1):268-277.

40. Abu-Amara M, Yang SY, Tapuria N, Fuller B, Davidson B, Seifalian A. Liver ischemia/reperfusion injury: processes in inflammatory networks--a review. Liver Transpl. 2010;16(9):1016-1032.

41. Abe Y, et al. Mouse model of liver ischemia and reperfusion injury: method for studying reactive oxygen and nitrogen metabolites in vivo. Free Radic Biol Med. 2009;46(1):1-7.

42. Zhang J, Dong Z, Zhou R, Luo D, Wei H, Tian Z. Isolation of lymphocytes and their innate immune characterizations from liver, intestine, lung and uterus. Cell Mol Immunol. 2005;2(4):271-280. 